\title{
Interiorização da Epidemia de COVID-19 na Região Nordeste: Existem Diferenças na Chance de Ocorrência dos Óbitos Segundo o Porte Populacional do Município?
}

\author{
The Spread of the COVID-19 Epidemic in Northeast Region, Brazil: are there \\ Differences in Risk of Death According to the City Size?
}

\section{RESUMO}

Objetivo: Analisar a expansão da epidemia de COVID-19 nos municípios de todos os estados da Região Nordeste do Brasil no mês de maio de 2020 e estimar, a partir dos dados dos boletins oficiais dos Estados, a chance de ocorrência de óbitos da COVID-19 em cada estado comparando municípios grandes com os de médio ou pequeno porte populacional. Metodologia: Trata-se de um estudo ecológico, espaço-temporal com avaliação em três momentos distintos entre os dias 7 e 22 do mês de maio. A expansão da epidemia foi descrita pelo percentual de municípios atingidos nos estados em cada momento no tempo e pelas chances de óbito, estimadas pela razão entre os casos de óbito registrados e o número de pessoas confirmadas com a doença, subtraído os óbitos. Resultados: Os resultados apontaram a interiorização acelerada da epidemia da COVID-19 na Região Nordeste e as chances de óbito diferentes estatisticamente em todos os estados, em pelo menos um dos períodos analisados. No Ceará, Maranhão e Paraíba as chances de óbito foram maiores nos municípios de grande porte populacional, com mais de 100 mil habitantes, enquanto nos demais estados da região as chances de óbitos foram maiores nos municípios de médio ou pequeno porte populacional. Conclusão: Os achados sugerem a ocorrência desigual da COVID-19 nos estados nordestinos ao comparar municípios de grande e médio porte populacional, o que alerta para a complexidade do processo epidêmico da COVID-19 em territórios com vulneráveis como o interior nordestino.

\section{DESCRITORES}

Infecções por Coronavirus. Disparidades nos Níveis de Saúde. Vulnerabilidade Social. Brasil.

\begin{abstract}
Objective: to analyze the expansion of the COVID-19 epidemic in the cities of all Northeastern States of Brazil during May 2020 and to estimate the chance of occurrence of deaths from COVID-19 comparing large and small/medium-sized cities in each State according to data from the official bulletins of the States' health departments. Methodology: This is a spatial-temporal ecological study that evaluated three time points from May 7 to 22. The epidemic spread was monitored by percentage of affected cities in each State over time. The chances of death from COVID-19 were estimated by the ratio between the number of confirmed deaths and the number of confirmed cases, subtracted by the number of deaths. Results: The results pointed to the rapid spread of the COVID-19 epidemic in the northeast region. We verified statistically significant differences of chance of death from COVID-19 in all States at least one of the three times analyzed. In Ceará, Maranhão and Paraíba the chance of death from COVID-19 was higher in populations who reside in large cities (more than 100 thousand inhabitants). While in other States of the Northeast the chances of death were higher in small or medium-sized cities. Conclusion: The findings suggest the unequal occurrence of COVID-19 due to the social inequalities and the disparities in access care, when comparing large and medium-sized cities, which warns of the complexity of the COVID-19 epidemic process in high social vulnerability territories.
\end{abstract}

\section{DESCRIPTORS}

Coronavirus Infections. Health Status Disparities. Social Vulnerability.

\footnotetext{
${ }^{1}$ Centro de Educação e Saúde, Universidade Federal de Campina Grande, Cuité, Paraíba Brasil

${ }^{2}$ Departamento de Nutrição, Universidade Federal da Paraíba, João Pessoa, Paraíba, Brasil.
} 
A COVID-19, doença causada pelo novo coronavírus (SARS-CoV-2), se espaIhou rapidamente ao redor do mundo. Cerca de 4 meses após a provável identificação do vírus na cidade de Wuhan, na China, a Organização Mundial de Saúde registrou cerca de 5 milhões de casos e mais de 300 mil mortes pela doença ${ }^{1}$. As consequências da pandemia de COVID-19 têm sido devastadoras para a população, pessoas infectadas, trabalhadores da saúde e a economia global em todos os continentes.

As pandemias, porém, não afetam todas as pessoas de maneira uniforme. Pesquisadores e organizações internacionais têm alertado para os riscos da expansão da epidemia de COVID-19 para áreas de alta vulnerabilidade social2-5. Populações pobres e em insegurança alimentar já enfrentam condições crônicas e problemas sociais que aumentam o risco de mortalidade associada à COVID-192, como também a menor capacidade de resiliência expõe de forma mais intensa populações e comunidades vulneráveis às consequências da crise econômica prevista em decorrência da pandemia ${ }^{6,7}$.

O Brasil é um território marcado pela grande desigualdade social, expressa na disparidade de renda e de acesso ao alimento de qualidade e em quantidade suficiente ${ }^{8,9}$. A desigualdade também se revela no acesso e na qualidade dos serviços de saúde ofertados pelo Sistema Único de Saúde (SUS) à população ${ }^{10-12}$. Essa diversidade de contextos e de estágios de desenvolvimento no território brasileiro ${ }^{13}$ coloca em debate as formas e os períodos distintos que a epidemia de COVID-19 vem impactando o Brasil, em face das dimensões continentais do país e os complexos e diversos arranjos sociais.

A experiência acumulada sobre o enfrentamento da pandemia aponta o maior risco de avanço da doença em regiões populosas e com menor adesão às medidas de distanciamento social ${ }^{14,15}$, como já observado nas grandes metrópoles e capitais brasileiras ${ }^{16}$. No entanto, apesar da menor densidade populacional, pesquisadores têm apontado a preocupação com a rápida expansão da epidemia para o interior do país, onde estão localizados municípios de médio ou pequeno porte populacional ${ }^{5,17}$. Esse alerta se deve tanto pela maior vulnerabilidade social existente nesses territórios, como pela menor capacidade resolutiva dos serviços de saúde instalados no interior do país para enfrentar a epidemia, de acordo com as diretrizes de regionalização dos serviços do SUS e a disponibilidade de recursos humanos e financeiros.

Em atenção a esse contexto de risco, a Região Nordeste é um dos territórios de maior vulnerabilidade social do Brasil. Cerca de $60 \%$ dos municípios nordestinos integram a área do semiárido brasileiro, considerada de extrema vulnerabilidade social e climáti$\mathrm{ca}^{20}$, caracterizada pela seca persistente e escassez de água, recurso essencial para a proteção individual contra a COVID-19. As condições climáticas e o insuficiente e inadequado investimento governamental criaram obstáculos históricos para o desenvolvimento social e econômico da região, especialmente entre a população rural ${ }^{21}$. Além disso, o Nordeste é a segunda região mais populosa do país, onde $96,8 \%$ e $66 \%$ dos municípios têm população estimada em menos de 100 e 20 mil habitantes, respectivamente. Tais municípios 
de pequeno porte populacional, especialmente localizados no interior, apresentam baixa capacidade político-administrativa ${ }^{18,19}$, e uma população majoritariamente dependente do SUS.

A hipótese levantada neste estudo considera que a expansão da epidemia da COVID-19 pelo interior da Região Nordeste implica em uma maior chance de óbitos entre a população residente em municípios de médio ou pequeno porte populacional, quando comparado com os municípios de grande porte. Desta forma, este estudo teve como objetivos (a) avaliar a expansão da COVID-19 nos municípios dos Estados da Região Nordeste (período de 7 a 21 de maio de 2020); e (b) analisar se existiu diferença na chance de ocorrência dos óbitos ao comparar a população residente em municípios de grande porte vs médio/pequeno porte populacional no mesmo período.

\section{METODOLOGIA}

Tipo de estudo e coleta de dados

Foi realizado um estudo ecológico ${ }^{22}$, com análise espaço temporal dos casos confirmados e óbitos por COVID-19, considerando o município de residência de cada caso proveniente dos estados da Região Nordeste do Brasil. Os dados agregados de cada estado foram estratificados pelo porte populacional do município.

A coleta de dados ocorreu em três etapas durante o período de 8 a 22 de maio deste ano, período de 15 dias em que ocorreu a confirmação de mais de 45 mil casos na região e mais de dois mil óbitos. A primeira etapa da coleta de dados (T1), ocorreu no dia 08 de maio de 2020, quando foram coletadas as notificações registradas até o dia 07 de maio para todos os estados, exceto o da $\mathrm{Pa}$ raíba cujos dados disponíveis se referem às notificações realizadas até o dia 02 de maio de 2020. A segunda etapa da coleta de dados (T2) ocorreu no dia 08 de maio de 2020, 7 dias após a primeira coleta e a terceira etapa (T3), no dia 22 de maio de 2020, 14 dias após o T1. Os dados foram coletados em números absolutos nas páginas da internet oficiais das Secretarias Estaduais de Saúde.

A divisão dos estratos dos municípios em dois grupos caracterizou-se da seguinte forma: (1) municípios com mais 100 mil habitantes, considerados de grande porte populacional (Alagoas: dois municípios; Bahia: 16 municípios; Ceará: nove municípios; Maranhão: oito municípios; Paraíba: quatro municípios; Pernambuco: 13 municípios; Piauí: dois municípios; Rio Grande do Norte: quatro municípios; Sergipe: quatro municípios) e (2) municípios com até 100 mil habitantes, considerados de médio e pequeno porte: demais municípios de cada Estado ${ }^{23}$.

Análise de dados

A expansão da doença em cada estado foi avaliada com base no percentual de municípios atingidos pela COVID-19, tendo em vista os dados coletados nas três etapas descritas ou tempos do estudo (T1, T2 e T3).

Foram estimadas as chances de óbito por meio da razão dos casos de óbito registrados com o número de casos confirmados da doença, subtraído os óbitos. Para comparar a chance de óbito por COVID-19 segundo o 
porte populacional do município foi calculada a Razão de Chances (RC), a partir da razão da chance de óbitos nos municípios de médio ou pequeno porte populacional (numerador) dividida pela chance de óbito dos municípios de grande porte populacional (denominador). Esta RC foi estimada em cada tempo do estudo (T1, T2 e T3) e para cada estado da região Nordeste ${ }^{24}$. O nível de significância de $95 \%$ foi adotado em toda a análise $(p<0,05)$. Os dados foram sistematizados e analisados no programa Excel for Windows e a razão de chances estimada no SciStat ${ }^{25}$.

Aspectos éticos

Não foi necessário submeter o estudo para aprovação do Comitê de Ética em Pesquisa, visto que os dados utilizados são de domínio público e sem identificação de participantes.

\section{RESULTADOS}

O percentual de municípios atingidos com casos confirmados de COVID-19 no T1 variou de $31,4 \%$ (Paraíba) a 90,1\% (Ceará). Em um período de 14 dias ocorreu um aumento no percentual de municípios com casos confirmados de COVID-19 em todos os estados: Paraíba (+ $37,2 \%$ ), Rio Grande do Norte (+31,1\%), Sergipe (+30,7\%), Maranhão $(+30 \%)$, Alagoas $(+29,4 \%)$, Piauí $(+20,1 \%)$, Pernambuco $(+17,8 \%)$, Bahia $(+17,3 \%)$ e Ceará $(+9,2 \%)$. No T3, o Estado do Ceará atingiu a marca de $100 \%$ dos municípios com casos confirmados da COVID-19 e o Estado da Bahia apresentou o menor percentual de expansão da doença, com $55,2 \%$ dos municípios atingidos (Gráfico 1). Variações significativas foram observadas na chance dos casos confirmados de COVID-19 evoluírem

Gráfico 1. Percentual de municípios com casos confirmados da COVID-19 segundo estado, Nordeste, Brasil, 2020

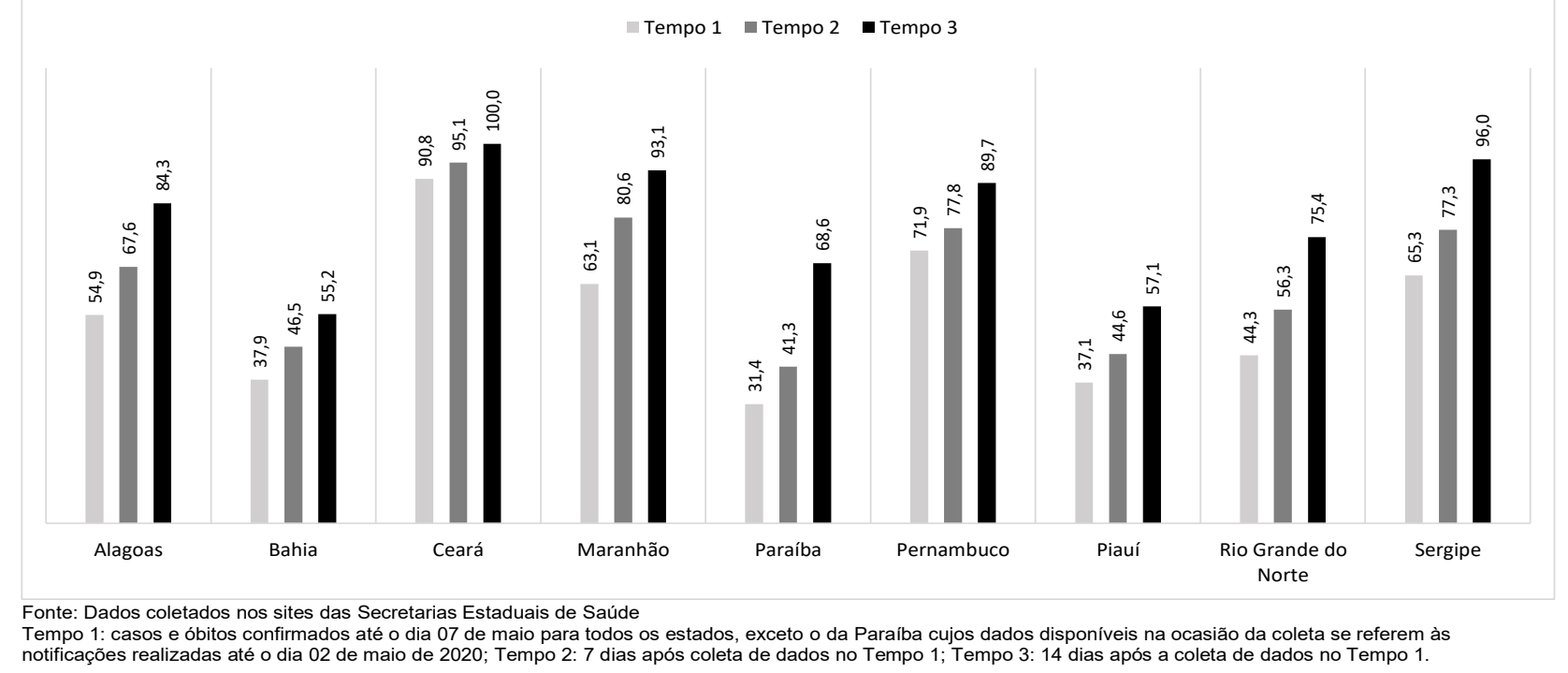


Tabela 1. Casos confirmados, óbitos, e razão de chances de óbitos por COVID-19 segundo estado e porte populacional dos municípios em estados da Região Nordeste, Brasil, maio de 2020

\begin{tabular}{|c|c|c|c|c|c|c|}
\hline \multirow[t]{2}{*}{ Estados } & \multirow{2}{*}{$T^{1}$} & \multicolumn{2}{|c|}{ Municipios grandes } & \multicolumn{2}{|c|}{$\begin{array}{l}\text { Municípios } \\
\text { pequenos ou } \\
\text { médios }\end{array}$} & \multirow{2}{*}{$\begin{array}{c}\text { Razão de chances } \\
(\mathrm{IC} \% 95)^{2}\end{array}$} \\
\hline & & $C(n)$ & $O(n)$ & $C(n)$ & $O(n)$ & \\
\hline \multirow{3}{*}{ Alagoas } & $\mathrm{T1}$ & 1512 & 66 & 355 & 32 & $2,17(1,40 ; 3,37)^{* *}$ \\
\hline & T2 & 2222 & 118 & 750 & 59 & $1,52(1,10 ; 2,11)$ * \\
\hline & T3 & 3297 & 176 & 1592 & 85 & $1,00(0,77 ; 1,31)$ \\
\hline \multirow{3}{*}{ Bahia } & T1 & 3826 & 131 & 702 & 34 & $1,44(0,98 ; 2,11)$ \\
\hline & $\mathrm{T} 2$ & 6029 & 210 & 926 & 52 & $1,65(1,21 ; 2,25)$ ** \\
\hline & T3 & 10034 & 302 & 1907 & 74 & $1,30(1,00 ; 1,69)$ * \\
\hline \multirow{3}{*}{ Ceará } & $\mathrm{T1}$ & 12109 & 865 & 3025 & 132 & $0,59(0,49 ; 0.71)^{\star \star \star \star}$ \\
\hline & $\mathrm{T} 2$ & 16878 & 1253 & 5612 & 223 & $0,52(0,45 ; 0,60)^{* * * *}$ \\
\hline & T3 & 22365 & 1782 & 9100 & 421 & $0,56(0,50 ; 0,63)^{* \star * \star}$ \\
\hline \multirow{3}{*}{ Maranhão } & T1 & 4671 & 298 & 1238 & 32 & $0,39(0,27 ; 0,56)^{* * \star}$ \\
\hline & $\mathrm{T} 2$ & 7017 & 436 & 3722 & 60 & $0,25(0,19 ; 0,32)$ *** \\
\hline & T3 & 9438 & 581 & 7774 & 112 & $0,22(0,18 ; 0,27)^{* * *}$ \\
\hline \multirow{3}{*}{ Paraíba } & T1 & 885 & 55 & 283 & 19 & $1,09(0,63 ; 1,86)$ \\
\hline & $\mathrm{T} 2$ & 1466 & 91 & 690 & 33 & $0,76(0,50 ; 1,14)$ \\
\hline & T3 & 2357 & 128 & 1706 & 55 & $0,58(0,42 ; 0,80)$ ** \\
\hline \multirow{3}{*}{ Pernambuco } & $\mathrm{T} 1$ & 9457 & 669 & 1367 & 176 & $1,94(1,63 ; 2,32)$ *** \\
\hline & $\mathrm{T} 2$ & 13570 & 1005 & 2018 & 293 & $2,12(1,85 ; 2,44)$ *** \\
\hline & T3 & 19826 & 1460 & 4085 & 465 & $1,62(1,45 ; 1,80)$ *** \\
\hline \multirow{3}{*}{ Piauí } & T1 & 761 & 21 & 370 & 16 & $1,59(0,82 ; 3,09)$ \\
\hline & $\mathrm{T} 2$ & 1194 & 30 & 711 & 30 & $1,71(0,72 ; 1,65)$ * \\
\hline & T3 & 1780 & 52 & 1294 & 41 & $1,09(0,72 ; 1,65)$ \\
\hline \multirow{3}{*}{$\begin{array}{l}\text { Rio Grande do } \\
\text { Norte }\end{array}$} & T1 & 1235 & 42 & 586 & 39 & $2,01(1,29 ; 3,15)$ ** \\
\hline & $\mathrm{T} 2$ & 1620 & 70 & 917 & 47 & $1,20(0,82 ; 1,75)$ \\
\hline & T3 & 2802 & 99 & 1258 & 79 & $1,83(1,35 ; 2,45)^{\text {** }}$ \\
\hline \multirow{3}{*}{ Sergipe } & T1 & 924 & 14 & 290 & 11 & $2,56(1,15 ; 5,71)^{*}$ \\
\hline & $\mathrm{T} 2$ & 1921 & 25 & 573 & 20 & $2,74(1,51 ; 4,98)$ ** \\
\hline & T3 & 3315 & 42 & 1419 & 34 & $1,91(1,21 ; 3,02)$ ** \\
\hline
\end{tabular}

Fonte: dados coletados nos sites das secretarias estaduais de saúde.

C: casos confirmados de COVID-19; O: óbitos confirmados por COVID-19 segundo município de residência.

1 T1: casos e óbitos confirmados até o dia 07 de maio para todos os estados, exceto Paraíba cujos dados disponíveis na ocasião da coleta se referem às notificações realizadas até o dia 02 de maio de 2020; T2: 7 dias após coleta de dados no T1; T3: 14 dias após a coleta de dados no T1. ${ }^{2}$ Calculada com base na população de casos confirmados em cada tempo de análise. ${ }^{*} \mathrm{P}<0,05$; ${ }^{* \star} \mathrm{P}<0,01$; $\mathrm{P}<0,001$. 
para óbito em função do porte populacional do município em todos os estados, em pelo menos um dos períodos analisados. No entanto, estas variações nem sempre tiveram a mesma direção. Os resultados observados apontam que existem dois cenários diferentes nos estados, o primeiro é onde a chance de óbito é maior nos municípios grandes, como foi o caso do Ceará, Maranhão e Paraíba. Nos demais estados a chance de óbito foi maior nos municípios de médio e pequeno porte populacional, em pelo menos um dos períodos analisados (Tabela 1, Gráfico 2). Os dados revelam mudanças nos três períodos analisados, com a redução progressiva da chance de óbito nos municípios de médio e pequeno porte populacional nos Estados de Alagoas, Maranhão, Paraíba, Piauí e Sergipe (Tabela 1, Gráfico 2).

\section{DISCUSSÃO}

O primeiro resultado deste estudo apontou a rápida expansão da COVID-19 para o interior dos estados nordestinos. Uma das principais preocupações já relatadas por pesquisadores e pelo governo brasileiro referente à interiorização da epidemia está no campo da capacidade dos serviços de saúde ${ }^{17}$. $\mathrm{O}$ deslocamento de pacientes que demandam assistência à saúde de média e alta complexidade para serviços de referência nos grandes centros urbanos pode sobrecarregar, ainda mais, o sistema de saúde pública, que apresentou sinais de colapso nessas cidades, além de ser um trabalho complexo o deslocamento, colocando em risco estes pacientes.

Para além dos impactos nos servi- ços de saúde, a interiorização da epidemia aponta para a exposição de comunidades socialmente e geograficamente vulneráveis às consequências devastadoras da epidemia. A Organização das Nações Unidas para Alimentação e Agricultura alertou que o número de subnutridos no mundo pode aumentar em até 80 milhões em decorrência da pandemia, especialmente nos países considerados de baixa renda ${ }^{7}$. Na mesma direção, o Banco Mundial estima a entrada de 2,7 milhões de pessoas da América Latina e Caribe na faixa de extrema pobreza em 2020, número expressivamente maior que o valor estimado para Europa/Ásia Central (0,9 milhões) e América do Norte $(0,1 \text { milhões })^{26}$.

Enquanto pesquisadores estimam a inaceitável previsão de 120 mil óbitos por COVID-19 no Brasil até o final do mês de agosto (dados de 27 de maio de 2020) ${ }^{35}$, o governo brasileiro demonstra incoerência e ineficiência na gestão da crise sanitária e de seus impactos na sociedade ${ }^{36}$, gerando um ambiente político e social regado de incertezas, insegurança e sensação de desamparo. No Brasil, a adoção de políticas de austeridade fiscal entre 2016-2018 e o alinhamento do atual presidente com tais medidas, conduziram a redução progressiva do investimento federal em políticas de saúde pública, educação e proteção social $^{27,28}$. De forma que, antes da epidemia da COVID-19, pesquisadores já alertavam para os riscos de aumento nas taxas de insegurança alimentar e pobreza na população brasileira, especialmente nos grupos vulneráveis ${ }^{29}$. A expansão da COVID-19 nos municípios nordestinos, neste cenário 
Gráfico 2. Aumento/diminuição, em porcentagem, de chance de óbito por COVID-19 segundo estado e porte populacional dos municípios em estados da Região Nordeste, Brasil. maio de 2020

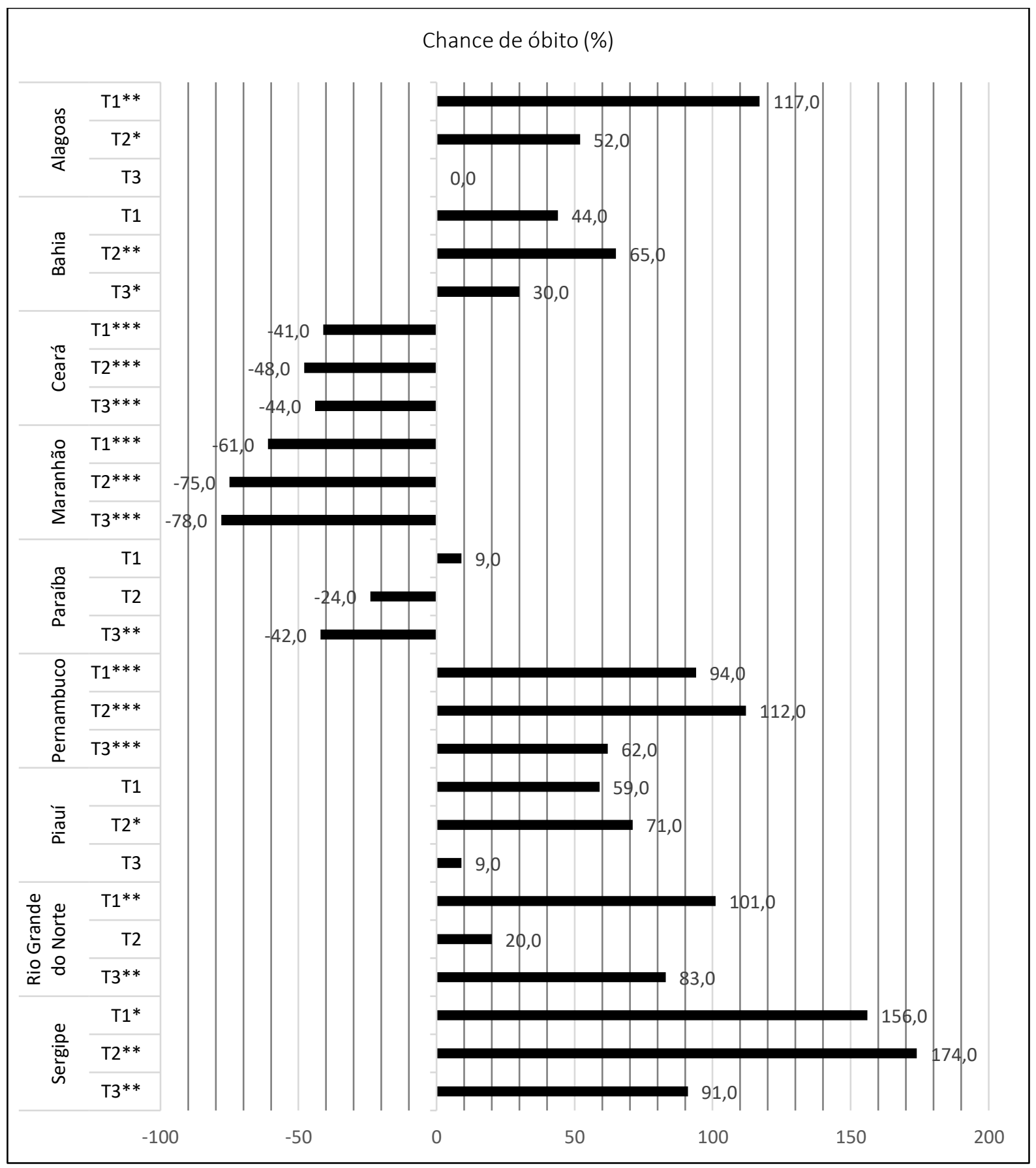

Fonte: dados coletados nos sites das secretarias estaduais de saúde.

T1: casos e óbitos confirmados até o dia 07 de maio para todos os estados, exceto Paraíba cujos dados disponíveis na ocasião da coleta se referem às notificações realizadas até o dia 02 de maio de 2020; T2: 7 dias após coleta de dados no T1; T3: 14 dias após a coleta de dados no T1. ${ }^{*} \mathrm{P}<0,001 ;{ }^{* *} \mathrm{P}<0,01 ;{ }^{* *} \mathrm{P}<0,05$. 
de desmonte de políticas públicas, sinaliza para o agravamento dos riscos sociais e da exposição da população à fome e à pobreza.

Outro objetivo deste estudo foi analisar a ocorrência de óbitos por COVID-19. Para os Estados de Alagoas, Bahia, Pernambuco, Piauí, Rio Grande do Norte e Sergipe a hipótese levantada neste estudo foi confirmada, ou seja, observou-se maior chance de evolução da doença para óbito na população acometida por COVID-19 residente nos municípios de médio e pequeno porte populacional, quando comparado aos municípios de grande porte. Nos estados do Ceará, Maranhão e Paraíba o resultado foi inverso.

Os casos existentes possibilitam avaliar a magnitude de uma doença na população, enquanto os óbitos sinalizam para a gravidade e o impacto. Diferenças na ocorrência de casos e óbitos são esperadas para populações com distintos perfis sociodemográficos e ambientais e de condições de acesso ao diagnóstico e ao tratamento da doença ${ }^{30}$. No contexto da epidemia da COVID-19 no Brasil, o alerta sobre a imprecisão dos dados oficiais disponíveis tem se tornado uma prerrogativa do processo da análise epidemiológica do fenômeno.

No caso do presente estudo, o desfecho analisado foi a ocorrência do óbito e a população estimada com base no número de casos de COVID-19 acumulados em um determinado período. Ambos os dados utilizados podem estar subnotificados por diferentes motivos: (1) reduzida capacidade de testagem, (2) direcionamento do diagnóstico para pacientes graves pela limitação da dis- ponibilidade de testes, (3) elevado número de óbitos com registro incorreto da causa base da morte.

A redução progressiva de chance de óbito nos municípios médios e pequenos observada em alguns estados confirma a ideia de que nestes municípios a subnotificação de casos é muito grande devido a limitação de testagem. Desta forma, no período observado, quando ocorreu expressivo aumento de casos confirmados e testagem, o que pode ter aumentando o denominador da base de cálculo da chance, reduzindo o seu valor. Considerando que este fenômeno aconteceu em todo o Brasil, chama a atenção que em outros estados, mesmo com o aumento do número de casos confirmados, devido o maior esforço de testagem, a chance de óbito não diminuiu, confirmando os riscos relacionados ao porte populacional municipal.

De fato, as grandes cidades apresentam um conjunto de características que elevam o risco de contaminação pela COVID-19, a exemplo do fluxo intenso de pessoas nas vias públicas, dos transportes públicos lotados e da existência de áreas periféricas e residenciais de baixa renda com elevada concentração de pessoas $^{31}$, sendo assim a implementação de medidas preventivas de distanciamento social são desafiadoras. Estes fatores justificam as maiores chances de óbito observadas nos municípios com maior porte populacional.

Por outro lado, tais contextos estão menos presentes nos municípios de pequeno e médio porte populacional, o que sugere menor taxa de transmissão e de acúmulo de casos. Nesta direção, um estudo em países africanos 
apontou que a existência de áreas escassamente povoadas, a exemplo das rurais, são fatores que contribuem para desacelerar a propagação da epidemia no continente ${ }^{32}$. No entanto, os autores não discutiram a letalidade por COVID-19 nessas regiões, ou seja, o possível impacto da vulnerabilidade social e da desigualdade no acesso à saúde na taxa de letalidade ainda é desconhecido.

No tocante à assistência à saúde, nas grandes cidades estão os serviços de alta complexidade e, portanto, maior disponibilidade de leitos, respiradores e profissionais de saúde, quando comparado aos municípios de pequeno e médio porte populacional ${ }^{17}$. Os municípios pequenos apresentam também outras fragilidades da assistência à saúde que se aplicam ao contexto da epidemia de COVID-19: (a) o acesso insuficiente aos testes rápidos; (b) a morosidade na confirmação dos casos suspeitos e na internação de pacientes graves; e (c) a limitação de recursos para o deslocamento dos pacientes para hospitais de referência fora do município. Estes fatores ajudam a explicar a maior chance de óbito em municípios de médio e pequeno porte populacional em alguns estados, como visto neste trabalho.

Diante disso, os limites da rede de atenção à saúde nas cidades de pequeno e médio porte, juntamente com a arrecadação pouco significativa e o incerto/tardio suporte financeiro do governo federal aos municípios, convergem para condições críticas relacionadas ao manejo da epidemia. Na ausência ou insuficiência de recursos para o diagnóstico e o tratamento da COVID-19, a implementação de medidas de prevenção e contenção precoce é a opção mais viável ${ }^{33}$, deve ser altamente recomendada e priorizada nos municípios de pequeno e médio porte populacional. $\mathrm{Na}$ grande maioria dos casos, como foi observada na evolução da epidemia nos municípios em todos os estados, esta oportunidade foi perdida por falta de planejamento e articulação das esferas estadual e federal de gestão da saúde. Para poucos municípios ainda é possível estabelecer estratégias preventivas de controle da transmissão da doença pessoa - pessoa para evitar a transmissão comunitária.

Governos estaduais decretaram medidas de controle da COVID-19 com enfoque no distanciamento social. Cabe aos gestores municipais implementar, monitorar e complementar tais medidas considerando a realidade local, sendo imprescindível o suporte técnico e financeiro dos governos estadual e federal. Além disso, é importante que os governos municipais, de forma integrada, tomem decisões regionalizadas para o controle da evolução da epidemia, considerando que existe uma relevante mobilidade da população entre municípios circunvizinhos, inclusive de estados diferentes.

\section{CONCLUSÃO}

Este estudo colocou em evidência uma das populações vulneráveis do Brasil e alerta para a rápida expansão da epidemia pelo interior do nordeste. Os resultados também sugerem a ocorrência desigual da COVID-19, em decorrência das desigualdades sociais e no acesso aos serviços de saúde. A interpre- 
tação dos resultados deve considerar que existe subnotificação de casos confirmados e de óbitos por COVID-19 no Brasil. A magnitude dessa subnotificação pode ser diferente entre os estados e os períodos analisados, o que impacta substancialmente no estimador calculado. Neste estudo não foram estimadas medidas ajustadas por eventuais variáveis de confusão, a exemplo da taxa de isolamento social e da capacidade de testagem dos estados nos três tempos analisados, sendo uma limitação da interpretação dos resultados. No entanto, a coleta e a análise dos dados em série temporal permitiram avaliar tendências relacionadas ao processo de interiorização da COVID-19, tema de grande relevância para atual fase da epidemia no Brasil, assim como mostrar que nos diferentes estados de uma mesma região a chance de óbito pode ser diferente em função do porte populacional do município e, consequentemente, de sua organização da assistência à saúde, orientando medidas diferentes de enfrentamento da epidemia.

Em um cenário em que não é possível estimar com precisão o número de casos e de óbitos, os resultados devem ser observados com muita cautela. Por outro lado, análises ex- ploratórias, como as realizadas neste estudo, contribuem para levantar novas hipóteses de direcionamento de ações de enfrentamento da epidemia. Do ponto de vista teórico, a análise realizada suscitou o debate sobre os diferentes contextos de risco para manejo da COVID-19 ao comparar municípios de grande e pequeno ou médio porte populacional.

Por fim, o enfrentamento da epidemia de COVID-19 demanda urgência visando proteger a população brasileira de suas consequências em curto e longo prazo. Nessa conjuntura, o processo epidêmico da COVID-19 no Brasil tem se mostrado cada vez mais complexo. Não se trata "apenas" da oferta suficiente de assistência à saúde, tarefa extremamente difícil e desafiadora, é necessário enfrentar a epidemia na perspectiva de reduzir, em curto tempo, também as desigualdades históricas que constrangem e violam o direito de todos à saúde. Assim, este estudo reforça a necessidade de implementar medidas preventivas e de tratamento para a COVID-19, que consideram diferenças existentes entre os municípios em termos de indicadores sociais e de estrutura dos serviços de saúde. 


\section{REFERÊNCIAS}

1. WHO. Coronavirus disease (COVID-19): WHO2020 Report No.: 123

2. Ahmed F, Ahmed Ne, Pissarides C, Stiglitz J. Why inequality could spread COVID-19. The Lancet Public Health. 2020; 5:e240.

3. Cornia GA, Richard J; Stewart, F. COVID-19 and children, in the North and in the South. Innocenti Discussion Papers, n 2020-02. Florence: UNICEF; 2020.

4. The Lancet Global H. Food insecurity will be the sting in the tail of COVID-19. The Lancet Global Health. 2020; 8:e737.

5. FIOCRUZ. Tendências atuais da pandemia de Covid-19 Interiorização e aceleração da transmissão em alguns estados. Rio de Janeiro, RJ: FIOCRUZ 2020 Contract No.: Nota técnica 28 de abril de 2020.

6. Laborde DD, Martin W, Vos R. Poverty and food insecurity could grow dramatically as COVID-19 spreads. Washington, DC: International Food Policy Research Institute, 2020.

7. Food and Organization of the United Nations. COVID-19 global economic recession: avoiding hunger must be at the centre of the economic stimulus. Rome: Food and Organization of the United Nations, 2020.

8. Instituto Brasileiro de Geografia e Estatística. Síntese de indicadores sociais : uma análise das condições de vida da população brasileira: 2019. Rio de Janiero, RJ: IBGE - Coordenação de População e Indicadores Sociais, 2019 Contract No.: 40.

9. Food and Organization of the United Nations. The State of Food Security and Nutrition in the World 2019. Safeguarding against economic slowdowns and downturns. Rome: Food and Organization of the United Nations, 2019.

10. Castro MC, Massuda A, Almeida G, Menezes-Filho NA Andrade MV, de Souza Noronha KVM, et al. Brazil's unified health system: the first 30 years and prospects for the future. The Lancet. 2019; 394.

11. Massuda A, Hone T, Leles FAG, de Castro MC, Atun R. The Brazilian health system at crossroads: progress, crisis and resilience. BMJ Global Health. 2018; 3:e000829.

12. Machado CV, Silva GAe. Political struggles for a universal health system in Brazil: successes and limits in the reduction of inequalities. Globalization and Health. 2019; 15:77.

13. Calvo MCM, Lacerda JTd, Colussi CF, Schneider IJC Rocha TAH. Estratificação de municípios brasileiros para avaliação de desempenho em saúde. Epidemiologia e Serviços de Saúde. 2016; 25:767-76.

14. Hellewell J, Abbott S, Gimma A, Bosse NI, Jarvis CI, Russell TW, et al. Feasibility of controlling COVID-19 outbreaks by isolation of cases and contacts. Lancet Glob Health. 2020; 8(4): e488 - e496.
15. Koo JR, Cook AR, Park M, Sun Y, Sun H, Lim JT, et al. Interventions to mitigate early spread of SARS-CoV-2 in Singapore: a modelling study. The Lancet Infectious Diseases. 2020; 20(6):678-688.

16. Covid-19: painel cororavírus [internet]. Brasília: Minitério da Saúde [acessado em: 25 de maio de 2020].

17. FIOCRUZ. Interiorização do Covid-19 e as redes de atendimento em saúde. Rio de janeiro: FIOCRUZ2020 02 de maio de 2020 Contract No.: Nota técnica 02 de maio de 2020.

18. Arretche M. Federalismo e políticas sociais no Brasil: problemas de coordenação e autonomia. São Paulo em Perspectiva 2004; 18:17-26.

19. Abrucio FL. A dinâmica federativa da educação brasileira: diagnóstico e propostas de aperfeiçoamento. In: Oliveira RP, Santana W, editors. Educação e federalismo no Brasil: combater as desigualdades, garantir a diversidade. Brasília: UNESCO; 2010. p. 39-70.

20. Instituto Brasileiro de Geografia e Estatística.Pesquisa Nacional por Amostra de Domicílios: Segurança Alimentar 2013. Rio de Janeiro: Instituto Brasileiro de Geografia e Estatística, Coordenação de trabalho e renda, 2014

21. Campos JN. Paradigms and Public Policies on Drought in Northeast Brazil: A Historical Perspective. Environmental Manegement. 2015; 55:12.

22. Medronho R. Estudos Ecológicos. In: Medronho R, Bloch K, Raggio R, Werneck G, editors. Epidemiologia. Rio de Janeiro: Atheneu; 2009. p. 265-74.

23. Instituto Brasileiro de Geografia e Estatística. Estimativas da população residente no Brasil e Unidades da Federação com data de referência em 1 de julho de 2019.

24. Kale P, Costa A, Raggio R. Medidas de associação e medidas de impacto. In: Medronho R, Bloch K, Raggio R, Werneck G, editors. Epidemiologia. Rio de Janeiro: Atheneu; 2009. p. 181-92.

25. MedCalc Software: SciStat [software]. 2020 [Acesso em 30 de junho de 2020].

26. Mahler D, Lakner C, Aguilar A, Wu H. The impact of COVID-19 (Coronavirus) on global poverty: Why Sub-Saharan Africa might be the region hardest hit [internet]. [Acessp em: 27 de maio de 2020]. Disponível em: https:// blogs.worldbank.org/opendata/impact-covid-19-coronavirus-global-poverty-why-sub-saharan-africa-might-be-region-hardest

27. Montenegro L, Velasque L, LeGrand S, Whetten K, Mattos R, Malta M. Public Health, HIV Care and Prevention, Human Rights and Democracy at a Crossroad in Brazil. AIDS and Behavior. 2020; 24:1-4.

28. Malta M. Human rights and political crisis in Brazil: Public health impacts and challenges. Global Public Health. 2018:1-8. 
29. Sousa LRMd, Segall-Corrêa AM, Ville AS, Melgar-Quiñonez $\mathrm{H}$. Food security status in times of financial and political crisis in Brazil. Cadernos de Saúde Pública. $2019 ; 35$.

30. Figueiredo A, Daponte A, Figueiredo DC, Gil-García E, Kalache A. Case fatality rate of COVID-19: absence of epidemiological pattern. Gac Sanit. 2020; Apr 4. S02139111(20)30084-4 (in press).

31. Burki T. COVID-19 in Latin America. The Lancet Infectious Diseases. 2020; 20:547-8.

32. Diop BZ, Ngom M, Pougué Biyong C, Pougué Biyong JN. The relatively young and rural population may limit the spread and severity of COVID-19 in Africa: a modelling study. BMJ Global Health. 2020; 5: e002699.

33. Bong CL, Brasher C, Chikumba E, McDougall R, Mellin-Olsen J, A. E. The COVID-19 Pandemic: Effects on Low and Middle-Income Countries. Anesth Analg. 2020; 131(1):86-92.
34. Pei S, Kandula S, Shaman J. Differential Effects of Intervention Timing on COVID-19 Spread in the United States. medRxiv [preprint]. May 2020; 2020.05.15.20103655.

35. IHME. COVID-19 Projections. Washington, USA: Institute for Health Metrics and Evaluation; 2020 [cited 202027 May 2020]; Available from: http://www.healthdata.org.

36. The L. COVID-19 in Brazil: "So what?". The Lancet. 2020; 395:1461.

\section{CORRESPONDÊNCIA}

Poliana de Araújo Palmeira

Sítio Olho d'agua da Bica, S/N,

Centro de Educação e Saúde, Cuité, Paraíba, Brasil CEP: $58175-000$

E-mail: palmeira.poliana@gmail.com 